\title{
The interaction of anticipatory anxiety and emotional picture processing: An event-related brain potential study
}

\author{
FLORIAN BUBLATZKY, TOBIAS FLAISCH, JESSICA STOCKBURGER, RALF SCHMÄLZLE, \\ AND HARALD T. SCHUPP \\ Department of Psychology, University of Konstanz, Konstanz, Germany
}

\begin{abstract}
The present study examined the interaction of anticipatory anxiety and selective emotion processing. Toward this end, a rapid stream of pleasant, neutral, and unpleasant pictures was presented in alternating blocks of threat-of-shock or safety, which were signaled by colored picture frames. The main finding is that pleasant pictures elicited a sustained negative difference potential over occipital regions during threat as compared to safety periods. In contrast, unpleasant and neutral picture processing did not vary as a function of threat-of-shock. Furthermore, in both the safety and threatof-shock conditions, emotional pictures elicited an enlarged early posterior negativity and late positive potential. These data show that the activation of the fear/anxiety network exerts valence-specific effects on affective picture processing. Pleasant stimuli mismatching the current state of anticipatory anxiety apparently draw more attentional resources.
\end{abstract}

Descriptors: Anxiety, Threat of Shock, Emotion, Attention, ERP

The perceptual processing of visual input is guided by stimulus significance. A large number of studies have consistently demonstrated a processing advantage for emotionally meaningful over neutral stimuli (Lang, Bradley, \& Cuthbert, 1997). The preferential processing of emotional stimuli may interact with attentional settings induced by the emotional state of the perceiver (Öhman, Flykt, \& Lundqvist, 2000; Pessoa, Kastner, \& Ungerleider, 2002; Schupp, Junghöfer, Weike, \& Hamm, 2003). Specifically, a state of fear/anxiety may result in enhanced attention toward emotionally significant stimuli. Merely telling individuals about imminent danger is sufficient to elicit a defensive motivational state in humans (Grillon, Ameli, Woods, Merikangas, \& Davis, 1991; Grillon \& Davis, 1995). Integrating both lines of research, the present study examined the effects of the threat-of-shock manipulation on the apparent attention capture by emotional pictures.

There is increasing evidence showing that pictures activating the appetitive or defensive motivation system guide selective attention processes (Lang et al., 1997; Öhman et al., 2000). Clear differences in autonomic, somatic, and reflex responses as a function of the valence and arousal of emotional pictures support

We are grateful to Beate Hofer for her assistance in data collection. This work was supported, in part, by the German Research Foundation (DFG) grants to H. T. Schupp (Schu1074/7-3, Schu1074/11-1) and a scholarship awarded to F. Bublatzky by the State Ministry for Education and Cultural Affairs of Baden-Wuerttemberg (LGFG).

Address reprint requests to: Florian Bublatzky, University of Konstanz, Department of Psychology, PO Box 36, 78457 Konstanz, Germany. E-mail: florian.bublatzky@uni-konstanz.de the concept of motivated attention (Bradley \& Lang, 2000; Lang $\&$ Davis, 2006). Furthermore, studies utilizing neuroimaging methods have demonstrated the enhanced perceptual processing of emotional cues. Functional magnetic resonance imaging (fMRI) studies revealed increased activations for emotionally arousing pictures in occipital, parietal, and inferior temporal cortices (Bradley et al., 2003; Junghöfer, Sabatinelli, et al., 2006; Junghöfer, Schupp, Stark, \& Vaitl, 2005; Sabatinelli, Bradley, Fitzsimmons, \& Lang, 2005). Event-related brain potential (ERP) studies revealed the temporal dynamics of visual attention to emotional stimuli. The processing of pleasant and unpleasant pictures as opposed to neutral images is consistently associated with an early posterior negativity (EPN) around $150-300 \mathrm{~ms}$ poststimulus (Junghöfer, Bradley, Elbert, \& Lang, 2001; Schupp et al., 2003). Subsequently, most apparent between 300 and $700 \mathrm{~ms}$ poststimulus, emotional pictures elicit an augmented late positive potential (LPP) over centro-parietal locations (Schupp et al., 2003, 2007). Specifying the relation to stimulus emotionality, both ERP components vary as a function of emotional arousal. In particular, the processing of highly arousing emotional pictures is associated with a more pronounced EPN and LPP compared to pictures of the same valence that are rated lower in arousal (Junghöfer et al., 2001; Schupp et al., 2000). According to a bivariate motivational model of emotion (Bradley \& Lang, 2000; Lang et al., 1997), enhanced attention to emotional cues supports the organization of efficient actions to stimuli (appetitive and defensive) that can sustain or threaten the life of the organism

Seen from an evolutionary perspective, learning about aversive events is critical in shaping defensive behaviors to avoid 
harm and danger to the organism (Öhman \& Mineka, 2001). In humans, a state of fear/anxiety can be elicited by social communication (Olsson \& Phelps, 2007). Merely the announcement about imminent danger is sufficient to elicit anticipatory anxiety in humans (Bradley, Moulder, \& Lang, 2005; Cornwell, Echiverri, Covington, \& Grillon, 2008; Funayama, Grillon, Davis, \& Phelps, 2001; Grillon et al., 1991; Grillon \& Davis, 1995). In the threat-of-shock paradigm, participants are verbally instructed that they might receive an electric shock when a specific cue is presented (e.g., red light) whereas another cue represents a safety period in which participants know they will not receive a shock. The activation of the defensive system in this protocol is revealed by a broad array of response measures, including self-report, behavior, and physiology. For instance, the difference between the threat-of-shock and safety conditions can be seen with potentiated startle reflexes, enlarged skin conductance responses, and cardiac deceleration (Bradley et al., 2005; Funayama et al., 2001; Grillon et al., 1991; Grillon \& Davis, 1995; Melzig, Weike, Zimmermann, \& Hamm, 2007). In addition, fMRI studies have revealed neural substrates of verbally induced expectations of aversive events. Threat-of-shock signals were found to be associated with increased BOLD activity in the amygdala, insular, and prefrontal cortices relative to safety signals (Dalton, Kalin, Grist, \& Davidson, 2005; Phelps et al., 2001). Further studies used event-related brain potentials to determine the modulation in cortical processing of previously neutral stimuli signaling either threat or safety conditions. Threat-of-shock increased several ERP components. These included early sensory components related to spatial frequency and later components such as the P3 wave, which has been suggested to be indicative of increased selective attention to stimuli cueing threat conditions (Baas, Kenemans, Böcker, \& Verbaten, 2002; Böcker, Baas, Kenemans, \& Verbaten, 2004). Overall, as with explicit fear conditioning studies (e.g., Davis, 1992; Öhman \& Mineka, 2001; Olsson \& Phelps, 2004, 2007), learning about aversive cues by means of verbal communication engages the defense system regulating perception, cognition, and avoidance behavior

The main goal of the present study was to explore the consequences of anticipatory anxiety on the preferential processing of emotionally significant stimuli. Accordingly, the research paradigms "threat-of-shock" and "picture viewing" were integrated by presenting emotional pictures during sustained threat-ofshock and safety conditions. In light of previous research, several hypotheses regarding the effects of threat-of-shock on picture processing can be derived. Recent studies have already shown that threat-of-shock modulates the processing of neutral stimuli at early sensory processing stages. For instance, brain stem wave $\mathrm{V}$, which is stimulated by simple click sounds and is held to be at least in part generated by the inferior colliculus, was larger when click sounds were presented during threat-of-shock than during safety periods (Baas, Milstein, Donlevy, \& Grillon, 2006). A possible implication of these findings is that threat-of-shock sensitizes perceptual picture processing. One may further postulate that threat-of-shock interacts with motivated attention processes, facilitating emotional picture processing. Regarding the effects of attention on emotion, a recent study observed that the late positive potential was enhanced when people attended to pictures of erotica or mutilations compared to neutral pictures (Schupp et al., 2007). Analogously, it might be posited that threat-ofshock effects are more pronounced for pleasant and unpleasant picture materials. According to this argument, the emotion-relevant context created by the threat-of-shock condition would

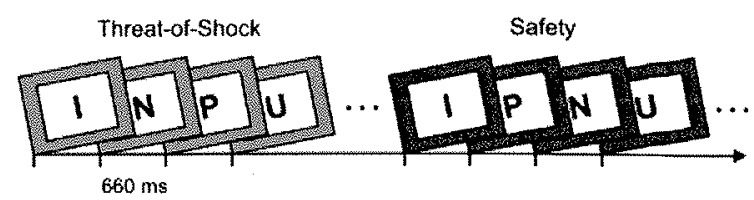

Figure 1. Schematic illustration of the experimental design. Emotional and neutral pictures were presented as a rapid and continuous picture stream (each $660 \mathrm{~ms}$ ). The participant were verbally instructed that the colored picture frames (blue and green) signaled either threat-of-shock or safety periods (assignment balanced across participants). Abbreviations $\mathrm{P}, \mathrm{N}, \mathrm{U}$ refer to pleasant, neutral, and unpleasant picture contents. I refers to instruction pictures announcing shock possible or no shock preceding each $40-s$ period of threat-of-shock or safety.

increase their significance. A different prediction is derived from a study in which pleasant and unpleasant pictures signaled either threat-of-shock or safety (Bradley et al., 2005). It was found that the presence of pleasant pictures cueing threat-of-shock potentiated startle reffexes and elicited autonomic responses consistent with defense activation. In contrast, unpleasant pictures did not show threat-of-shock-induced startle potentiation. Thus, the congruity or incongruity of threat-of-shock with the hedonic picture valence may produce distinct effects on perceptual processing of pleasant and unpleasant picture cues.

The present study aimed to examine the effects of threat-ofshock on emotional and neutral picture processing. Toward this end, pleasant, neutral, and unpleasant pictures were presented $(660 \mathrm{~ms})$ in random order as a rapid and continuous serial stream while colored background picture frames signaled periods of threat-of-shock and safety, each lasting $\sim 40 \mathrm{~s}$ (see Figure 1). In line with previous studies, participants were instructed that they might receive aversive but nonpainful electric shocks during the threat-of-shock condition. Two streams of analyses were conducted to examine whether threat-of-shock-induced sensitization effects are similar across picture contents, differ as a function of stimulus valence (pleasant vs. neutral vs. unpleasant), or reflect stimulus arousal (pleasant/unpleasant vs. neutral). One set of analyses served to replicate previous findings regarding the emotional modulation of the EPN and LPP components and explored whether these differences are modulated by threat-ofshock. Furthermore, rather than modulating the obligatory sequence of ERP components, a sustained activation of the defense system may induce distinct effects on the processing of emotional and neutral pictures. Thus, in a further set of analyses, single sensor ERP waveform and conventional analyses based on sensor clusters and time windows were used to assess differences among the threat-of-shock and safety conditions and their interaction with picture valence.

\section{Methods}

\section{Participants}

Participants were 16 healthy students ( 8 women) between the ages of 20 and 35 years $(M=24.4)$ recruited from the University of Konstanz (Beck Depression Inventory $M=3.5, S D=2.2$; STAI-State $M=36, S D=3.8$; STAI-Trait $M=35, S D=5.4$ ). Before providing written informed consent, participants were fully informed about the study protocol, which was approved by the institutional ethics committee. Participants received 10 Euros for their participation. 


\section{Stimulus Materials and Presentation}

Sixty pictures were selected from the International Affective Picture System (IAPS; Lang, Bradley, \& Cuthbert, 2008) depicting naturalistic scenes of neutral (e.g., humans in nonemotional everyday life situations), pleasant (e.g., erotica, sports), and unpleasant (e.g., mutilation, human and animal threat) contents. Highly arousing pleasant and unpleasant picture contents were selected, as these materials elicit most pronounced modulations in ERP, FMRI, defensive reflex, and autonomic measures (cf. Bradley, Codispoti, Cuthbert, \& Lang, 2001; Junghöfer et al., 2001, 2005; Schupp, Flaisch, Stockburger, \& Junghöfer, 2006; Schupp et al., 2003, 2004).

The IAPS pictures $(640 \times 480$ pixels $)$ were surrounded by a colored picture frame $(1024 \times 768$ pixels), which indicated conditions (threat-of-shock vs. safety). Experimental conditions were signaled by either blue or green frame color, which was adjusted for brightness. Across participants, frame colors signaling the threat or safety condition were balanced. Furthermore, an instruction picture ("shock possible" or "no shock") preceded each experimental block.

Stimuli were presented as a rapid and continuous stream without interstimulus gap. Across the picture stream (see Figure 1), blocks of the threat-of-shock and safety conditions (blue or green frames) were presented alternately. The starting condition, threat-of-shock or safety, was balanced across participants. During each of the 30 blocks, pleasant, neutral, and unpleasant pictures were shown for $660 \mathrm{~ms}$ in random order with no more than three repetitions of the same picture category allowed. Each participant viewed a different order of IAPS picture presentation. The full set of pictures $(N=60)$ was presented during each block. The entire picture set was repeated 30 times for a total of 1800 trials. Pictures were presented on a 22 -in. computer screen with a refresh rate of $85 \mathrm{~Hz}$. Distance between participants and the screen was approximately $75 \mathrm{~cm}$.

\section{Shock Workup Procedure}

A shock workup procedure ensured the credibility of the threatof-shock instruction (cf. Bradley et al., 2005). Preceding the main experiment, participants received up to eight shocks. The intensity of the electric shocks increased from below threshold $(0.3$ $\mathrm{mA}$ ) until the participants reported the stimulation as "maximal unpleasant, but not painful." Afterward, participants were told that the intensity of the electric shocks given during the experiment would be equal to the most unpleasant test stimulus. Electrical stimuli with a maximum intensity of $10 \mathrm{~mA}$ (duration 10 $\mathrm{ms}$ ) were administered using a constant current electro stimulator applied through a stimulation electrode ( $1 \mathrm{~mm}$ in diameter) at the tip of the left index finger. No shocks were delivered during the experiment itself. This was to avoid sensitization effects associated with shock delivery (Greenwald, Bradley, Cuthbert, \& Lang, 1998) and because sustained and robust threat-of-shock effects can be produced by mere verbal instructions (Grillon et al., 1991; Grillon \& Davis, 1995).

The IAPS picture numbers were as follows: pleasant, 1610,1710 , $1722,1750,2070,2080,4232,4290,4460,4550,4601,4606,4610,4611$, $4651,4652,4670,4680,4690,4700$ - neutral $1620,1670,1720,1812$ $2200,2210,2372,2381,2383,2394,2440,2485,2499,2510,2530,2580$, $2850,4605,5410,5875$; unpleasant, 0013, 1300, 1525, 1930, 3010, 3015, $3061,3063,3064,3071,3102,3110,3120,3130,6250,6313,6550,6560$, 6570,9584 .

\section{Procedure}

All participants were given a brief description of the procedure prior to the study and completed several questionnaires (Beck Depression Inventory, STAI-State, and STAI-Trait) to screen for participants with extreme scores on anxiety and depression scales. Participants with psychiatric or neurological illnesses were excluded from participation as well. After the attachment of the electroencephalogram (EEG) sensor net, participants were seated in a dimly lit and sound-attenuated room. During a practice run, participants were familiarized with the picture viewing paradigm and instructed to attend to each picture appearing on the screen. Afterward, the shock electrode was attached and the shock workup procedure was performed. Subsequently, participants were told that they may receive electric shocks during picture viewing and instructed about the assignment of frame color to either the threat-of-shock or safety condition. During a break in the middle and at the end of the experiment, participants reported their emotional experience during the threat-of-shock and safety conditions. Valence and arousal ratings were obtained using the self-assessment manikin (SAM; Bradley \& Lang, 1994), and the perceived threat was rated on a visual analog scale (range $0-10$ ). At the end of the experiment, IAPS pictures were also evaluated on valence and arousal dimensions.

\section{EEG Recording}

Electrophysiological data were collected from the scalp using a 257-lead geodesic sensor net (Electrical Geodesics, Inc. [EGI], Eugene, OR). The EEG was recorded continuously with a sampling rate of $250 \mathrm{~Hz}$ with the vertex sensor as reference electrode, and online filtered from 0.1 to $100 \mathrm{~Hz}$ using Netstation acquisition software and EGI amplifiers. Impedances were kept below $30 \mathrm{k} \Omega$, as recommended for this type of amplifier by EGI guidelines. Off-line analyses were performed using EMEGS (Junghöfer \& Peyk, 2004) including low-pass filtering at $30 \mathrm{~Hz}$, artifact detection, sensor interpolation, baseline correction, and conversion to an average reference (Junghöfer, Elbert, Tucker, \& Rockstroh, 2000; Junghöfer, Peyk, Flaisch, \& Schupp, 2006). Stimulus-synchronized epochs were extracted lasting from 100 $\mathrm{ms}$ before to $660 \mathrm{~ms}$ after stimulus onset. Finally, separate average waveforms were calculated for the six experimental cells (three picture categories $\times$ threat-of-shock and safety conditions) for each sensor and participant.

\section{Data Reduction and Analyses}

Self-report data. To confirm expected differences between the threat-of-shock and safety conditions, separate $t$ tests were calculated for valence, arousal, and threat ratings. Similarly, to replicate previous findings regarding the IAPS picture ratings, repeated measures analyses of variances (ANOVAs) including the factor Picture Category (pleasant, neutral, unpleasant) were calculated.

Event-related potentials. To reveal effects of threat-of-shock on picture processing and to determine corresponding sensor clusters and time windows, visual inspection and single sensor waveform analyses were used in concert. For the waveform analyses, ANOVAs containing the factors Threat-of-Shock (threat, safety) and Picture Category (pleasant, neutral, unpleasant) were calculated for each time point after picture onset separately for each individual sensor (cf. Schupp et al., 2003).

In the main analyses, repeated measures ANOVAs based on mean activity in selected sensor clusters and time windows were 
performed. Mean amplitudes from bilateral posterior sensor clusters (EGI sensor numbers: left 105, 106, 107, 113, 114, 115, $116,117,121,122,123,124,125,126,134,135,136,137,146$, 147 ; right $139,149,150,151,157,158,159,160,161,166,167$, $168,169,175,176,170,177,178,188,189)$ were averaged for five time intervals ranging from 80 to $580 \mathrm{~ms}$. Data were entered into a four-factorial ANOVA including the factors Threat of Shock (threat, safe), Picture Category (pleasant, neutral, unpleasant), Laterality (left, right), and Time (five intervals: 80-180, 180-280, $280-380,380-480,480-580 \mathrm{~ms}$ ).

To explore threat-of-shock effects on emotion processing, the EPN and LPP were analyzed as in previous research (Schupp et al., 2006). The EPN component was scored in bilateral posterior sensor clusters (see above) in a time window from 140 to $280 \mathrm{~ms}$. The LPP was scored as mean activity in bilateral centro-parietal sensor clusters (EGI sensor numbers: left $9,17,42,43,44,51,52$, $59,77,78,79,87,88,99$; right $130,131,132,133,143,144,145$, $155,156,185,186,187,198,199)$ in a time window from 400 to $600 \mathrm{~ms}$. Data were entered into repeated measures ANOVAs including Threat of Shock, Picture Category, and Laterality.

For effects involving repeated measures, the GreenhouseGeisser procedure was used to correct for violations of sphericity. To control for Type 1 error, Bonferroni correction was applied for post hoc tests.

\section{Results}

\section{Self-Report Data}

Threat ratings. Threat-of-shock sequences were rated significantly more threatening than safety periods $(M=4.2$ and 1.4 , $S D=2.1$ and 1.6$), t(15)=6.00, p<.001$. In addition, the threatof-shock condition was perceived as more unpleasant and higher in arousal compared to the safety condition, $t \mathrm{~s}(15)=2.32$ and $3.48, p<.05$ and $p<.01$, respectively (threat-of-shock: $M_{\text {valence }}=4.3, S D=1.2, M_{\text {arousial }}=4.6, S D=1.6$, and safety: $M_{\text {valence }}=5.6, S D=1.8, M_{\text {arousial }}=3.1, S D=1.5$ ).

Picture ratings. Valence and arousal ratings differed among picture categories, $F \mathrm{~s}(2,30)=164.23$ and $43.98, p s<.001, \varepsilon=.95$ and .75 , respectively. ${ }^{2}$ Pleasant pictures were evaluated more positively $(M=6.8, S D=0.7)$ compared to neutral images $(M=5.8, S D=0.6), p<.01)$ and aversive pictures $(M=2.3$, $S D=0.7)$ were judged as more unpleasant than neutral contents, $p<.001$. Regarding arousal reports, pleasant and unpleasant picture categories $(M=3.3$ and $5.5, S D=1.1$ and 1.9) were rated as more arousing as compared to neutral cues $(M=2.3$, $S D=1.0), p<.05$ and $p<.001$. In addition, unpleasant pictures were evaluated as more arousing compared to pleasant cues, $p<.001$.

\section{Event-Related Potentials}

Threat-of-shock effects are illustrated in Figure 2 separately for pleasant, neutral, and unpleasant picture contents. As can be seen, the threat-of-shock compared to the safety condition affected primarily the processing of pleasant picture cues. During threat-of-shock, pleasant picture processing was associated with a sustained negativity over temporo-occipital regions and a cor-

${ }^{2}$ The IAPS ratings corresponded very closely to normative ratings obtained for these pictures. The Pearson correlation analyses between the sample and normative ratings were .95 for valence and .80 for arousal dimension.
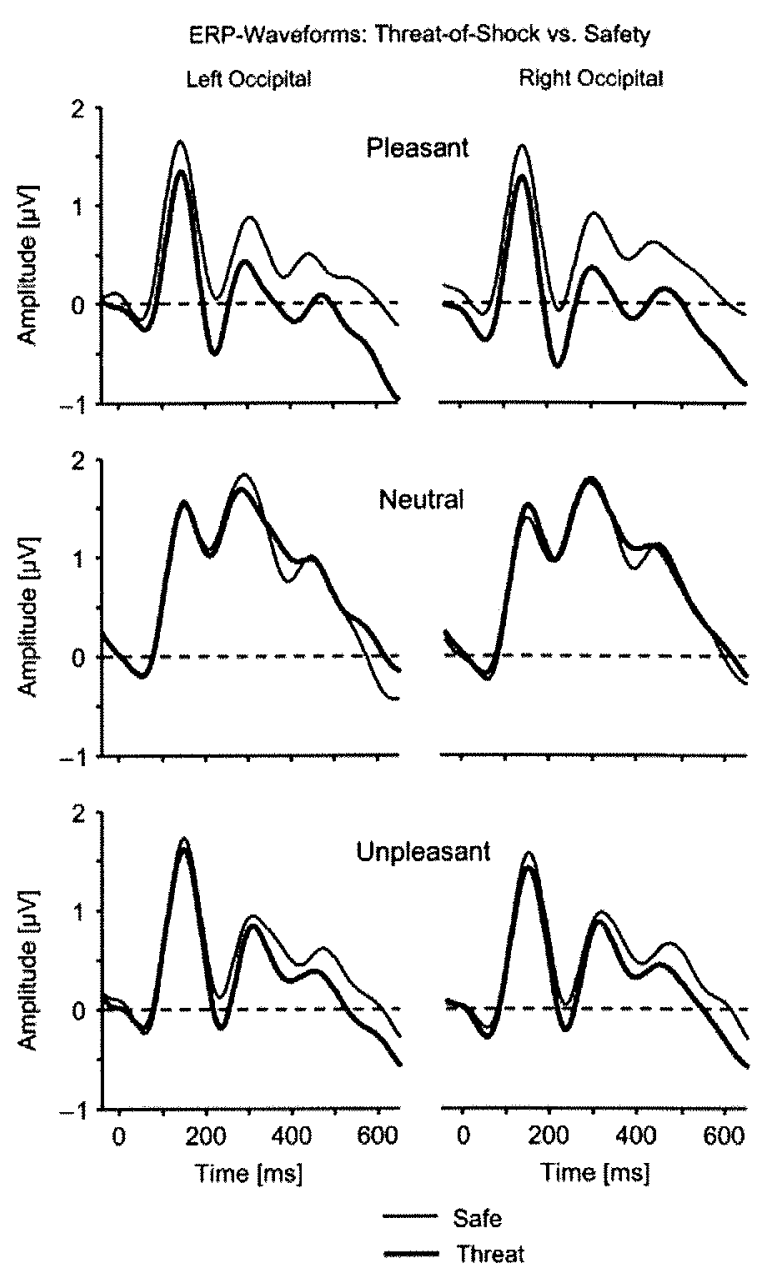

Figure 2. Grand-averaged ERP waveforms of a representative left and right occipital sensor as a function of picture valence and context conditions (threat-of-shock or safety).

responding polarity reversal over centro-frontal sites, which appeared around $80-100 \mathrm{~ms}$ poststimulus and lasted for several hundred milliseconds (see Figures 2 and 3). Furthermore, emotional differentiation indicated by the EPN and LPP was replicated in the safety condition and similarly present during the threat-of-shock condition. Specifically, as shown in Figures 4 and 5 , pleasant and unpleasant pictures were associated with enhanced EPN and LPP amplitudes compared to neutral contents in both conditions. To substantiate these findings, the sustained posterior negativity and emotion-sensitive ERP components (EPN and LPP) were explored in separate ANOVAs.

\section{Sustained Posterior Negativity}

Statistical analysis revealed significant main effects of Threat of Shock, $\quad F(1,15)=7.60, \quad p<.05, \quad$ Picture Category, $F(2,30)=12.06, p<.001, \varepsilon=.93$, and Time, $F(4,60)=4.77$, $p<.05, \varepsilon=.59$. No hemispheric differences were observed, $F(1,15)=0.50, p=.49$. Of most interest, the main effect of Threat of Shock was qualified by the higher-order interaction Threat of Shock $\times$ Picture Category, $F(2,30)=4.36, p<.05$, 


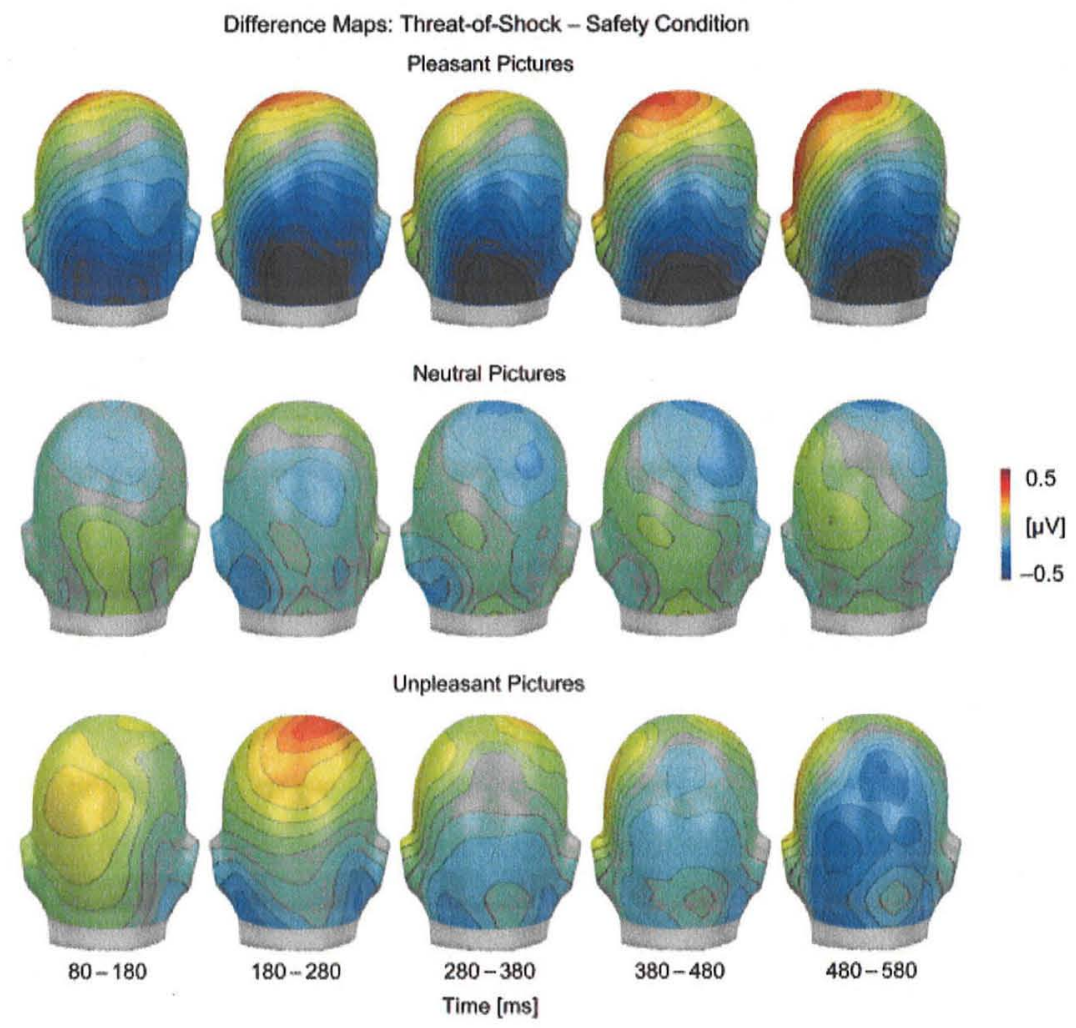

Figure 3. Topographical difference maps (threat-of-shock - safety condition) separately for pleasant, neutral, and unpleasant picture contents. The maps display averaged time intervals plotted on the back view of a model head.

$\varepsilon=.93$. To follow up this interaction, separate ANOVAs were calculated for pleasant, unpleasant, and neutral picture contents including the factors Threat of Shock, Laterality, and Time.

Pleasant pictures. The presentation of pleasant pictures during threat-of-shock conditions was associated with a sustained posterior negativity compared to safety conditions, Threat of Shock $F(1,15)=12.03, p<.01$. This effect lasted for several hundred milliseconds, as indicated by the nonsignificant interaction of Threat of Shock $\times$ Time, $F(4,60)=0.43, p=.71$, $\varepsilon=.65$. For exploratory reasons, the threat-of-shock effect was analyzed separately for each time interval. As shown in Table 1, the threat-of-shock-induced posterior negativity for pleasant pictures during threat compared to safety conditions was significant for each 100-ms time interval from 80 to $580 \mathrm{~ms}$ poststimulus, Threat of Shock $F \mathrm{~s}(1,15)=8.65,9.64,9.45,10.70$, and $9.43, p s<.02$.

The only other effect reaching significance in this analysis was Time, $F(4,60)=6.82, p<.01, \varepsilon=.60$, indicating a decrease in amplitude over time.

Unpleasant pictures. Whereas inspection of Figure 2 suggests an enhanced sustained posterior negativity for unpleasant pictures during the threat-of-shock compared to safety conditions, neither the main effect of Threat of Shock $F(1,15)=0.90$, $p=.36$, nor the higher-order interaction Threat of Shock $\times$ Time reached significance, $F(4,60)=0.59, p=.60, \varepsilon=.65$. Exploratory analyses of separate time intervals (from 80 to $580 \mathrm{~ms}$ poststimulus) revealed no significant threat-of-shock effects,
Threat of Shock $F \mathrm{~s}(1,15)=0.02,1.23,1.12,0.45$, and 0.88 , $p s=.88, .28, .31, .51$, and .36

Similar to pleasant picture processing, the main effect Time approached significance, $F(4,60)=3.09, p=.06, \varepsilon=.53$, indicating a decrease in amplitude over time.

Neutral pictures. Neutral picture processing was not modulated by threat-of-shock. Neither the main effect Threat of Shock, $F(1,15)=0.04, p=.84$, nor the higher-order interaction Threat of Shock $\times$ Time were statistically significant, $F(4,60)=0.42, p=.72, \varepsilon=.65$, and exploratory tests for each time interval did not approach significance, Threat of Shock, $F \mathrm{~s}(1,15)=0.21,0.30,0.001,0.30$, and $0.04, p \mathrm{~s}=.66, .59, .98$, .59 , and .85 .

Again, over the course of picture presentation time the amplitude decreased significantly, $F(4,60)=9.41, p<.001, \varepsilon=.52$.

\section{Early Posterior Negativity}

Replicating previous findings, the EPN amplitude varied as a function of Picture Category, $F(2,30)=20.82, p<.001, \varepsilon=.73$. Follow-up tests revealed that pleasant and unpleasant picture processing were associated with enlarged EPN amplitudes compared to neutral cues, $F(1,15)=59.44$ and $11.51, p s<.01$, respectively. As in previous research, the EPN to pleasant pictures was enlarged compared to that to unpleasant images, $F(1,15)=5.63, p<.05$.

Consistent with the analysis of the sustained posterior negativity ( $80-580-\mathrm{ms}$ time windows), the EPN (140-280 ms) revealed a significant main effect of Threat of Shock, 
A ERP-Waveforms: Early Posterior Negativity

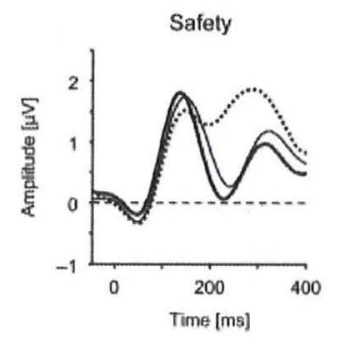

Threat-of-Shock

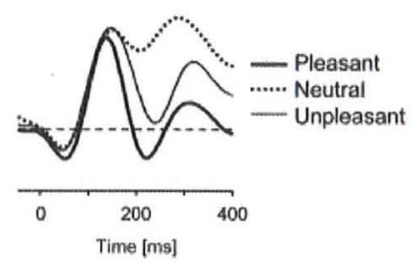

B

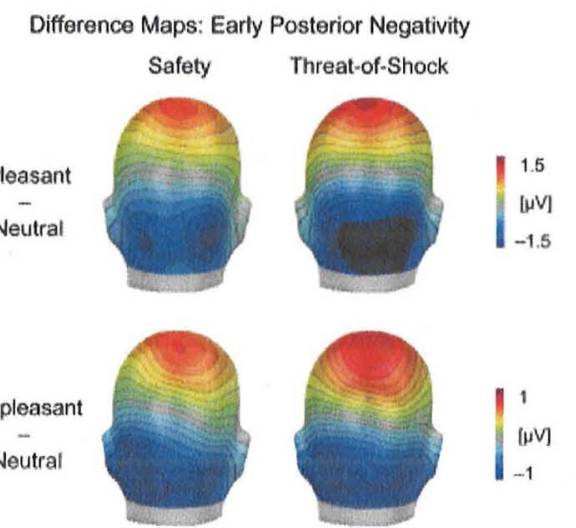

Figure 4. A: Grand-averaged waveforms (right occipital sensor) of the three picture categories illustrating the EPN during safety (left) and threat-of-shock (right) conditions. B: Topographical difference maps of the EPN for pleasant - neutral and unpleasant - neutral pictures as a function of context (safety and threat-of-shock). Maps display the back view of a model head (means from 140 to $280 \mathrm{~ms}$ ).

$F(1,15)=7.53, p<.05$, and an interaction of Threat of Shock $x$ Picture Category, $F(2,30)=3.63, p<.05, \varepsilon=.98$. Comparing the threat-of-shock and safety for each picture category separately revealed an enlarged EPN during the threat-of-shock condition for pleasant picture processing, $F(1,15)=10.22, p<.01$, whereas no significant threat-of-shock effects were observed for unpleasant and neutral images, $F \mathrm{~s}(1,15)=0.83$ and $.01, p \mathrm{~s}=.38$ and .92 , respectively. In addition, separate analyses of the threatof-shock and safety conditions confirmed the overall analysis, Picture Category, $F \mathrm{~s}(2,30)=20.05$ and $13.06, p s<.001, \varepsilon s=.72$ and .91, respectively, revealing significantly enlarged EPN during pleasant and unpleasant compared to neutral picture processing for threat $F \mathrm{~s}(1,15)=70.20$ and $9.12, p s<.01$, and safety condition, $F \mathrm{~s}(1,15)=24.95$ and $11.38, p \mathrm{~s}<.01$.

\section{Late Positive Potential}

Similar to previous studies, the LPP varied as a function of Picture Category, $F(2,30)=26.92, p<.001, \varepsilon=.92$. Pleasant and unpleasant pictures elicited larger LPPs compared to neutral materials, $F \mathrm{~s}(1,15)=43.81$ and $35.68, p<.001$, respectively. In addition, the LPP was comparable for both emotional picture categories, $F(1,15)=0.98, p=.34$.

Neither the main effect of Threat of Shock, $F(1,15)=0.0002$, $p=.99$, nor the higher-order interaction of Threat of shock $\times$ Picture Category, $F(2,30)=0.79, p=.46, \varepsilon=.97$, approached significance.
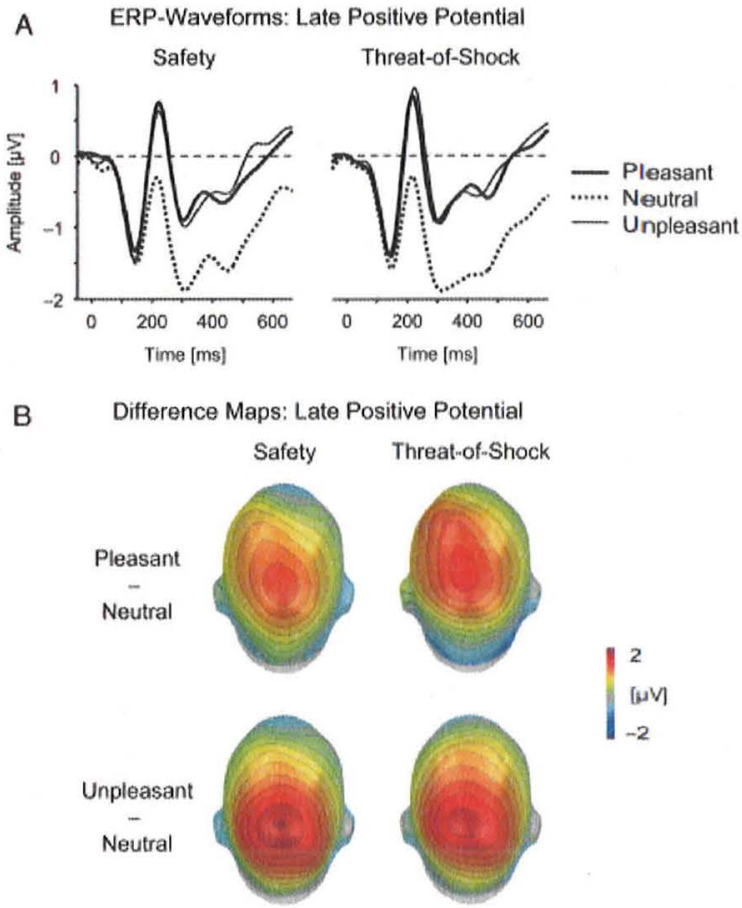

Figure 5. A: Grand-averaged waveforms (right parietal sensor) of the three picture categories illustrating the LPP during safety (left) and threat-of-shock (right) conditions. B: Topographical difference maps of the LPP for pleasant - neutral and unpleasant - neutral pictures as a function of context (safety and threat-of-shock). Maps display the top view of a model head (means from 400 to $600 \mathrm{~ms}$ ).

\section{Habituation Effects}

Repeatedly experiencing threat-of-shock and safety conditions might prompt habituation effects. Previous studies found that self-reported distress as well as potentiated startle responses were relatively sustained across repetitions of threat and safety cues (Grillon et al., 1991; Mol, Baas, Grillon, van Ooijen, \& Kenemans, 2007). Given that habituation effects may vary across

Table 1. Threat of Shock by Picture Category and Time Interval

\begin{tabular}{|c|c|c|c|c|c|c|}
\hline \multirow{2}{*}{$\begin{array}{l}\text { Picture } \\
\text { category }\end{array}$} & \multirow[b]{2}{*}{ Conditions } & \multicolumn{5}{|c|}{ Time intervals (ms) } \\
\hline & & $80-180$ & $180-280$ & $280-380$ & $380-480$ & $480-580$ \\
\hline \multirow[t]{4}{*}{ Pleasant } & Threat & $0.84^{*}$ & $-0.07^{* * *}$ & $0.28 * *$ & $-0.00^{* *}$ & $-0.21^{\text {*** }}$ \\
\hline & & $(0.59)$ & $(1.06)$ & $(0.78)$ & $(0.87)$ & $(0.96)$ \\
\hline & Safe & 1.19 & 0.38 & 0.72 & 0.48 & 0.25 \\
\hline & & $(0.68)$ & $(0.90)$ & $(0.71)$ & $(0.72)$ & $(0.66)$ \\
\hline \multirow{4}{*}{ Neutral } & Threat & 1.09 & 1.41 & 1.54 & 1.04 & 0.53 \\
\hline & & $(0.60)$ & $(0.76)$ & $(0.86)$ & $(0.77)$ & $(0.71)$ \\
\hline & Safe & 1.05 & 1.44 & 1.54 & 0.98 & 0.51 \\
\hline & & $(0.51)$ & $(0.78)$ & $(0.86)$ & $(0.72)$ & $(0.74)$ \\
\hline \multirow[t]{4}{*}{ Unpleasant } & Threat & 1.13 & 0.42 & 0.83 & 0.58 & 0.28 \\
\hline & & $(0.71)$ & $(1.58)$ & $(0.79)$ & $(0.83)$ & $(0.82)$ \\
\hline & Safe & 1.14 & 0.57 & 0.97 & 0.69 & 0.47 \\
\hline & & $(0.63)$ & $(1.35)$ & $(0.67)$ & $(0.76)$ & $(0.88)$ \\
\hline
\end{tabular}

Note: Mean amplitudes $(S D)$ for pleasant, neutral, and unpleasant pictures during the threat-of-shock and safety conditions averaged over occipital sensor clusters and time intervals from 80 to $580 \mathrm{~ms}$ (data in microvolts).

$* p<.05 ; * * *<<.01$. 
different measures and experimental procedures, complementary control analyses were conducted including the additional factor Session (first vs. second half) to address habituation effects.

Regarding threat ratings, a significant interaction of Threat of Shock $\times$ Session was observed, $F(1,15)=6.00, p<.05$. Separate post hoc analysis of the threat-of-shock condition revealed that perceived threat decreased from the first to the second half of the experiment ( $M=5.1$ and $3.3, S D=2.8$ and 2.2 , respectively), $p<.01$. In contrast, threat ratings did not vary across time within the safety condition $(M=1.6$ and $1.3, S D=1.8$ and 1.7$)$, $p=1.0$. However, differences between threat and safety conditions were highly significant regarding both the first, $p<.001$, and the second half of the experiment, $p<.01$, respectively. No significant interactions of Threat of Shock $\times$ Session were observed for valence and arousal ratings, $F \mathrm{~s}(1,15)=0.88$ and 2.54 , $p s=.36$ and .13 , respectively.

Regarding the analysis of the sustained posterior negativity, neither the main effect of Session, $F(1,15)=1.35, p=.26$, nor the interaction of Threat of Shock $\times$ Session, $F(1,15)=0.57$, $p=.46$, nor the three-way interaction $\times$ Picture Category reached significance, $F(2,30)=0.26, p=.73, \varepsilon=.82$. Whereas the separate analysis for pleasant pictures revealed no interaction of Threat of Shock $\times$ Session, $F(1,15)=0.35, p=.56$, exploratory analyses showed that the effect of an enhanced sustained posterior negativity for pleasant pictures during the threat-ofshock compared to safety conditions was somewhat more pronounced during the first than the second half of the experiment, first half $F(1,15)=5.08, p<.05$, second half $F(1,15)=3.70$, $p=.07$. For unpleasant and neutral pictures neither Threat of Shock $\times$ Session, $F \mathrm{~s}(1,15)=0.12$ and $0.57, p s=.73$ and .46 , nor exploratory analyses separately for first and second halves of the experiment revealed significant effects, unpleasant $F \mathrm{~s}(1,15)=0.22$ and $0.52, \quad p s=.64$ and .48 , neutral $F \mathrm{~s}(1,15)=0.18$ and $0.60, p s=.68$ and .45 , respectively.

As in previous research (Codispoti, Ferrari, \& Bradley, 2006, 2007; Schupp et al., 2006), affective EPN and LPP modulation did not vary over the course of time. Neither the main effect of Session, $F(1,15)=0.44, p=.52$, nor the interaction of Session $\times$ Picture Category reached significance for the EPN component, $F(2,30)=0.09, p=.87, \varepsilon=.80$. Similarly, for the LPP, neither the main effect of Session, $F(1,15)=2.44, p=.14$, nor the interaction of Session $\times$ Picture Category was significant, $F(2,30)=0.35, p=.70, \varepsilon=.99$.

\section{Discussion}

The present study explored the consequences of threat-of-shock on the preferential processing of emotional pictures. Participants were told that they might receive aversive electrical shocks at unpredictable times during threat-of-shock periods. The anticipation of aversive events modulated the processing of concurrently presented pleasant pictures. Specifically, during the threatof-shock, pleasant pictures were associated with a sustained posterior negativity. This effect appeared rather early in the visual processing stream $(\sim 80-100 \mathrm{~ms})$ and lasted for several hundred milliseconds. In contrast, processing of unpleasant and neutral pictures did not significantly vary as a function of context conditions (threat-of-shock vs. safety). These findings provide a first demonstration of valence-specific changes in perceptual processing through anticipatory anxiety, suggesting an emotional mismatch principle of anxiety state and stimulus valence

Key findings for both picture viewing and threat-of-shock paradigms were replicated. Regarding perceptual picture processing, the safety condition allowed the comparison of the selective processing of emotional and neutral pictures in a nonthreatening context. As a replication of previous findings (Schupp et al., 2006), pleasant and unpleasant pictures elicited larger EPN and LPP amplitudes than neutral images. Furthermore, merely telling participants about the possibility of receiving electric shocks, even when no shock is actually administered, is highly effective and reliable in activating the defense system in humans (Baas, Grillon, et al., 2002; Grillon et al., 1991, 1999; Grillon \& Baas, 2003). Likewise, in the present study, participants consistently evaluated the threat-of-shock periods as more threatening, aversive, and emotionally arousing than the safety periods. Establishing these findings provided the necessary foundation to meaningfully interpret the effects of threat-of-shock on emotional picture processing.

Anatomical and neuroimaging studies suggest a neural base for the interaction of anticipatory anxiety and visual emotion processing. An interconnected network of cortical and subcortical limbic structures is thought to regulate the preferential processing of emotional stimuli (Vuilleumier, 2005). A key structure is the amygdala, which has direct and indirect routes modulating visual cortical processing (Amaral, Behniea, \& Kelly, 2003; Lang \& Davis, 2006). For instance, there is evidence that increased amygdala activation is correlated with the enhanced processing of fearful face stimuli in the fusiform face area (Vuilleumier, Armony, Driver, \& Dolan, 2001) and patients with lesions in mediotemporal cortex failed to show enhanced activity in the fusiform gyrus (Vuilleumier, Richardson, Armony, Driver, \& Dolan, 2004). Neuroimaging studies revealed that the transient activation of the human defense system by threat-of-shock has at least in part a similar neural structure including the activation of the amygdala, insular, and extended regions in the prefrontal cortex (Dalton et al., 2005; Phelps et al., 2001). Building upon these findings, the present study provided first empirical evidence that the engagement of the human defensive system through anticipation of unpredictable electric shocks modulates the processing of natural complex pictures. Moreover, the present study reveals important boundary conditions determining the interaction of the stimulus-driven visual attention and emotional state. First, the activation of the human defensive system specifically modulated the perceptual processing of appetitive pictures. Second, threat-of-shock effects appeared as a distinct ERP modulation, rather than affecting emotion-sensitive ERP components (EPN and LPP).

During threat-of-shock periods, appetitive picture processing was associated with a sustained negative difference potential over occipital regions. Negative-going ERP components have been observed in various domains of cognitive neuroscience. Research in the field of attention, memory, and language suggests distinct negative ERP components (e.g,, mismatch negativity, N2, selection negativity, N400), which are commonly considered to reflect facilitated stimulus processing (Kutas \& Federmeier, 2000; Näätänen, 1995; Potts \& Tucker, 2001). Furthermore, mental rotation, associative learning, and anticipation tasks elicit sustained negative slow waves, which are considered to reflect attentional resources in task-related processing modules (Rösler, Heil, Bajric, Pauls, \& Hennighausen, 1995; Rösler, Heil, \& Röder, 1997). On the basis of these findings, the sustained 
negativity over visual processing areas elicited by pleasant pictures in the threat-of-shock context might reflect more elaborate stimulus processing and increased allocation of attentional resources. Regarding the antecedent conditions, the presentation of stimuli evoking a physical or semantic mismatch to the current context is associated with negative ERP components (MMN and N400; Kutas \& Federmeier, 2000; Näätänen, 1995). Analogously, the incongruity between a pleasant foreground picture and the threat-of-shock context may explain the current findings in terms of an emotional mismatch. This hypothesis would predict facilitated perceptual processing of aversive pictures during appetitive states. Accordingly, future studies in which pleasant and unpleasant pictures are presented during comparable activation of the appetitive and aversive motivational systems would be pertinent (cf. Löw, Lang, Smith, \& Bradley, 2008).

A noteworthy aspect of the present results concerns the early onset $(\sim 80-100 \mathrm{~ms})$ of the threat-of-shock manipulation on pleasant picture processing. For this to occur specifically to pleasant but not unpleasant or neutral pictures, hedonic valence of the pictures had to be extracted early in the visual processing stream. Previous studies with IAPS picture materials and facial expressions reported emotion-related ERP differences around $120-150 \mathrm{~ms}$ after stimulus onset (Holmes, Vuilleumier, \& Eimer, 2003; Junghöfer et al., 2001; Schupp et al., 2004, 2008). Rather than revealing emotional differentiation, these early onset differences could be attributed to differences in low-level physical stimulus characteristics (e.g., spatial frequency, color, complexity) among emotional and neutral stimulus categories. For instance, Van Rullen \& Thorpe (2001) observed early ERP differences (75-80 ms) as a function of stimulus category (animal vs. vehicle). Similarly, IAPS pictures varying in figureground composition and spatial frequency were associated with different ERP potentials between 150 and $250 \mathrm{~ms}$ (Bradley, Hamby, Löw, \& Lang, 2007). However, in the present study, differences in low-level physical stimulus characteristics were excluded by comparing identical picture materials presented in both experimental context conditions (threat-of-shock and safety). Moreover, the observed onset latency of emotion discrimination is similar to the modulation of early stages of visual processing shown in fear-conditioning ERP studies (Pizzagalli, Greischar, \& Davidson, 2003; Stolarova, Keil, \& Moratti, 2006). Overall, the current findings suggest a considerably earlier onset latency of the extraction of emotional meaning than previous studies.

The current findings did not support several alternative hypotheses regarding the interaction of emotional state and preferential emotion processing. Unpleasant and neutral pictures were processed similarly when viewed during threat-of-shock and safety conditions. Furthermore, habituation analyses substantiated these findings in showing similar effects for the first and second halves of the experiment. Thus, neither the hypotheses that threat-of-shock sensitizes the processing of emotionally arousing pictures (pleasant and unpleasant) nor a general sensitization account (irrespective of picture valence) was supported. Furthermore, the data provided no support for a motivational priming account, which would predict that sustained defensive activation particularly sensitizes the processing of unpleasant pictures. Although Figure 2 suggests a somewhat pronounced pattern of sustained posterior negativity for unpleasant pictures during threat-of-shock, this effect failed to approach statistical significance. Future studies need to assess whether these findings extend beyond the specific implementation of picture presenta- tion. For instance, inducing mood states by blocked presentations of pleasant, neutral, and unpleasant pictures might reveal motivational priming effects (Bradley, Cuthbert, \& Lang, 1996; Pastor et al., 2008; Smith, Löw, Bradley, \& Lang, 2006). Furthermore, it is possible that the current findings can be explained through the alternation of threat-of-shock and safety blocks. Specifically, a between-subject design might have provided a more powerful manipulation of emotional state and possibly different effects on picture processing. A recent study, though with significant methodological differences from the present study, also suggested that threat-of-shock effects were limited to pleasant picture materials. In this study, pleasant and unpleasant pictures signaled threat-of-shock and safety conditions whereas the startle reflex served as a measure of defensive engagement (Bradley et al., 2005). The startle reflex was augmented when pleasant pictures signaled a threat-of-shock rather than safety. In contrast, no significant difference between threat-of-shock and safety conditions occurred for unpleasant picture cues (Bradley et al., 2005). According to these findings, emotional incongruity seems to be of importance when one considers the interaction of emotional state and picture valence.

The finding that neutral stimulus processing was not affected by the threat-of-shock manipulation contrasts with previous studies assessing obligatory ERP components. Specifically, the auditory brain stem wave $\mathrm{V}$ and the mismatch negativity were shown to be enhanced when simple click sounds were presented in a threat-of-shock condition instead of in safety periods (Baas et al,, 2006; Cornwell et al., 2007). However, methodological differences (e.g., visual vs. auditory stimulus modality; complex vs. simple stimuli) may account for the divergent findings. Furthermore, the present study was not designed to probe obligatory ERP components (e.g., brain stem waves or MMN). Thus, awaiting more conclusive evidence, it seems possible that using less complex visual stimulus materials reveals the modulation of neutral stimulus processing by threat-of-shock. These caveats notwithstanding, threat-of-shock manipulation did not exert a general sensitization effect on the processing of naturalistic complex pictures.

The present study provided little evidence that threat-ofshock specifically modulated emotion-sensitive ERP components. This finding is unlikely to be the result of using a serial presentation paradigm. Previous research revealed robust extraction of emotional meaning at much higher presentation rates (Peyk, Schupp, Keil, Elbert, \& Junghöfer, 2009), similar to cognitive research providing evidence for ultrarapid picture categorization based on higher-order semantic meaning (Codispoti, Ferrari, Junghöfer, \& Schupp, 2006; Thorpe, Fize, \& Marlot, 1996). Furthermore, emotional EPN and LPP modulation was consistent with previous research utilizing rapid serial picture presentation (cf.' Flaisch, Junghöfer, Bradley, Schupp, \& Lang, 2008). Regarding the LPP, enhanced amplitudes to pleasant and unpleasant pictures in comparison to neutral pictures were similarly pronounced in the threat-of-shock and safety periods. Considering the LPP as a measure of stimulus relevance and selective attention (Nieuwenhuis, Aston-Jones, \& Cohen, 2005; Schupp et al., 2006), threat-of-shock potentiated the LPP of neither unpleasant nor pleasant pictures. A somewhat different pattern was observed for the EPN time window, which revealed enhanced negativity for pleasant pictures during threat-ofshock. However, this significant modulation appears to reflect the sustained posterior negativity effect spanning a much longer period of time rather than the specific enhancement of the EPN 
component. These findings imply different consequences of threat-of-shock and explicit selective attention manipulations on emotion-sensitive ERP components. A recent study in which participants were asked to pay attention to emotional cues provided evidence of additive and synergistic effects of explicit attention on the EPN and LPP components, respectively (Schupp et al., 2007). Thus, explicit attention and threat-of-shock manipulations have distinct effects on emotion-sensitive ERP components (Schupp et al., 2006).

In summary, the present study provides evidence for the interaction of anticipatory anxiety and visual attention to emotion.
The anticipation of aversive events altered the processing of specifically appetitive picture cues. During threat-of-shock periods, pleasant pictures elicited a sustained posterior negativity, which appeared comparatively early in the processing stream (80-100 ms) and lasted for several hundred milliseconds. Thus, emotional stimuli that do not match the current state of anticipatory anxiety seem to draw more attentional resources. Conversely, stimulus processing of unpleasant and neutral pictures was not affected by threat-of-shock. These findings suggest that the incongruity between emotional state and stimulus valence modulates stimulusdriven attention capture.

\section{REFERENCES}

Amaral, D. G., Behniea, H., \& Kelly, J. L. (2003). Topographic organization of projections from the amaygdala to the visual cortex in the macaque monkey. Neuroscience, 1/8, 1099-1120

Baas, J. M., Grillon, C., Böcker, K. B., Brack, A. A., Morgan, C. A. 3rd, Kenemans, J. L., et al. (2002). Benzodiazepines have no effect on fearpotentiated startle in humans. Psychopharmacology, 161, 233-247.

Baas, J. M., Kenemans, J. L., Böcker, K. B., \& Verbaten, M. N. (2002). Threat-induced cortical processing and startle potentiation. NeuroReport, 13, 133-137.

Baas, J. M., Milstein, J., Donlevy, M., \& Grillon, C. (2006). Brainstem correlates of defensive states in humans. Biological Psychiatry, 59, $588-593$.

Böcker, K. B., Baas, J. M., Kenemans, J. L., \& Verbaten, M. N. (2004). Differences in startle modulation during instructed threat and selective attention. Biological Psychology, 67, 343-358.

Bradley, M. M., Codispoti, M., Cuthbert, B. N., \& Lang, P. J. (2001). Emotion and motivation I: Defensive and appetitive reactions in picture processing. Emotion, 1, 276-298.

Bradley, M. M., Cuthbert, B. N., \& Lang, P. J. (1996). Picture media and emotion: Effects of a sustained affective context. Psychophysiology, $33,662-670$

Bradley, M. M., Hamby, S., Löw, A., \& Lang, P. J. (2007). Brain potentials in perception: Picture complexity and emotional arousal Psychophysiology, 44, 364-373.

Bradley, M. M., \& Lang, P. J. (1994). Measuring emotion: The SelfAssessment Manikin and the Semantic Differential. Journal of Behavior Therapy and Experimental Psychiatry, 25, 49-59.

Bradley, M. M., \& Lang, P. J. (2000). Measuring emotion: Behavior, feeling, and physiology. In R. D. Lane \& L. Nadel (Eds.), Cognitive neuroscience of emotion (pp. 242-276). New York: Oxford University Press.

Bradley, M. M., Moulder, B., \& Lang, P. J. (2005). When good things go bad: The reflex physiology of defense. Psychological Science, 16, 468473

Bradley, M. M., Sabatinelli, D., Lang, P. J., Fitzsimmons, J. R., King, W., \& Desai, P. (2003). Activation of the visual cortex in motivated attention. Behavioral Neuroscience, 117, 369-380.

Codispoti, M., Ferrari, V., \& Bradley, M. M. (2006). Repetitive picture processing: Autonomic and cortical correlates. Brain Research, 1068, 213-220

Codispoti, M., Ferrari, V., \& Bradley, M. M. (2007). Repetition and event-related potentials: Distinguishing early and late processes in affective picture perception. Journal of Cognitive Neuroscience, 19 , $577-586$.

Codispoti, M., Ferrari, V., Junghöfer, M., \& Schupp, H. T. (2006). The categorization of natural scenes: Brain attention networks revealed by dense sensor ERPS. Neurolmage, 32, 583-591.

Conwell, B. R., Baas, J. M., Johnson, L., Holroyd, T., Carver, F. W. Lissek, S., et al. (2007). Neural responses to auditory stimulus deviance under threat of electric shock revealed by spatially-filtered magnetoencephalography. Neurolmage, 37, 282-289.

Cornwell, B. R., Echiverri, A. M., Covington, M. F., \& Grillon, C. (2008). Modality-specific attention under imminent but not remote threat-of-shock. Psychological Science, 19, 615-622.

Dalton, K. M., Kalin, N. H., Grist, T. M., \& Davidson, R. J. (2005). Neural-cardiac coupling in threat-evoked anxiety. Joumal of Cognilive Neuroscience, 17, 969-980.
Davis, M. (1992). The role of the amygdala in fear and anxiety. Annual Review of Neuroscience, 15, 353-375.

Flaisch, T., Junghöfer, M., Bradley, M. M., Schupp, H. T., \& Lang, P. J (2008). Rapid picture processing: Affective primes and targets Psychophysiology, 45, 1-10.

Funayama, E. S., Grillon, C., Davis, M., \& Phelps, E. A. (2001). A double dissociation in the affective modulation of startle in humans: Effects of unilateral temporal lobectomy. Joumal of Cognitive Neuroscience, 13, 721-729.

Greenwald, M. K., Bradley, M. M., Cuthbert, B. N., \& Lang, P. J. (1998). Startle potentiation: Shock sensitization, aversive learning and affective picture modulation. Behavioral Neuroscience, 112 1069-1079.

Grillon, C., Ameli, R., Woods, S. W., Merikangas, K., \& Davis, M. (1991). Fear-potentiated startle in humans: Effects of anticipatory anxiety on the acoustic blink reflex. Psychophysiology, 28, 588-595.

Grillon, C., \& Baas, J. (2003). A review of the modulation of the startle reflex by affective states and its application in psychiatry. Clinical Neurophysiology, 114, 1557-1579.

Grillon, C., \& Davis, M. (1995). Acoustic startle and anticipatory anxiety in humans: Effects of monaural right and left ear stimulation. Psychophysiology, 32, I55-161.

Grillon, C., Merikangas, K. R., Dierker, L., Snidman, N., Arriaga, R. I. Kagan, J., et al. (1999). Startle potentiation by threat of aversive stimuli and darkness in adolescents: A multi-site study. International Joumal of Psychophysiology, 32, 63-73.

Holmes, A Vuilleumier, P. \& Eimer, M. (2003). The processing of emotional facial expression is gated by spatial attention: Evidence from event-related brain potentials. Cognitive Brain Research, 16 , 174-184.

Junghöfer, M., Bradley, M. M., Elbert, T. R., \& Lang, P. J. (2001) Fleeting images: A new look at early emotion discrimination. Psychophysiology, 38, 175-178.

Junghöfer, M., Elbert, T., Tucker, D. M., \& Rockstroh, B. (2000) Statistical control of artifacts in dense array EEG/MEG studies. Psychophysiology, 37, 523-532.

Junghöfer, M., \& Peyk, P. (2004). Analyse und Visualisierung von Hirnstrom- und Hirnmagnetfeld-Messungen. [Analysis of electrical potentials magnetic fields of the brain]. Matlab Select, 2, 24-28.

Junghöfer, M., Peyk, P., Flaisch, T., \& Schupp, H. T. (2006). Neuroimaging methods in affective neuroscience: Selected methodologica issues. Progress in Brain Research, 156, 123-143.

Junghöfer, M., Sabatinelli, D., Bradley, M. M., Schupp, H. T., Elbert, T R., \& Lang, P. J. (2006). Fleeting images: Rapid affect discrimination in the visual cortex. NeuroReport, 17, 225-229.

Junghöfer, M., Schupp, H. T., Stark, R., \& Vaitl, D. (2005). Neuroimaging of emotion: Empirical effects of proportional global signal scaling in $\mathrm{fMRI}$ data analysis. Neurolmage, 25, 520-526.

Kutas, M., \& Federmeier, K. D. (2000). Electrophysiology reveals semantic memory use in language comprehension. Trends in Cognitive Sciences, 4, 463-470

Lang, P. J., Bradley, M. M., \& Cuthbert, B. N. (1997). Motivated attention: Affect, activation, and action. In P. J. Lang, R. F. Simons, \& M. Balaban (Eds.), Attention and emotion: Sensory and motivational processes (pp. 97-135). Mahwah, NJ: Erlbaum.

Lang, P. J., Bradley, M. M., \& Cuthbert, B. N. (2008). International affective picture system (IAPS): Affective ratings of pictures and in- 
struction manual. Technical Report A-8. Gainesville, FL: University of Florida.

Lang, P. J., \& Davis, M. (2006). Emotion, motivation, and the brain: Reflex foundations in animal and human research. Progress in Brain Research, 156, 3-29.

Löw, A., Lang, P. J., Snith, J. C., \& Bradley, M. M. (2008). Both predator and prey: Emotional arousal in threat and reward. Psycho. logical Science, 19, 865-873.

Melzig, C. A., Weike, A. I., Zimmermann, J., \& Hamm, A. O. (2007). Startle reflex modulation and autonomic responding during anxious apprehension in panic disorder patients. Psychophysiology, 44, 846854.

Mol, N., Baas, J. M. P., Grillon, C., van Ooijen, L., \& Kenemans, J. L. (2007). Startle potentiation in rapidly alternating conditions of high and low predictability of threat. Biological Psychology, 76, 43-51.

Näätänen, R. (1995). The mismatch negativity: A powerful tool for cognitive neuroscience. Ear \& Hearing, 16, 6-18

Nieuwenhuis, S., Aston-Jones, G., \& Cohen, J. D. (2005). Decision making, the P3, and the locus coeruleus-norepinephrine system. Psychological Bulletin, 131, 510-532.

Öhman, A., Flykt, A., \& Lundqvist, D. (2000). Unconscious emotion: Evolutionary perspectives, psychophysiological data and neuropsychological mechanisms. In R. D. Lane \& L. Nadel (Eds.), Cognitive neuroscience of emotion (pp. 296-327). New York: Oxford University Press.

Öhman, A., \& Mineka, S. (2001). Fears, phobias, and prepardness: Toward an evolved module of fear and fear learning. Psychological Review, $108,483-522$

Olsson, A. \& Phelps, E. A. (2004). Learned fear of "unseen" faces after Pavlovian, observational, and instructed fear. Psychological Science, $15,822-828$

Olsson, A., \& Phelps, E. A. (2007). Social learning of fear. Nature Neuroscience, I0, 1095-1102

Pastor, M. C., Bradley, M. M., Löw, A., Versace, F., Moltó, J., \& Lang, P. J. (2008). Affective picture perception: Emotion, context, and the late positive potential. Brain Research, 1189, 145-151.

Pessoa, L., Kastner, S., \& Ungerleider, L. G. (2002). Attentional control of the processing of neutral and emotional stimuli. Cognitive Brain Research, 15, 31-45.

Peyk, P., Schupp, H. T., Keil, A., Elbert, T., \& Junghöfer, M. (2009). Parallel processing of affective visual stimuli. Psychophysiology, 46, 200-208.

Phelps, E. A., O'Connor, K. J., Gatenby, J. C., Gore, J. C., Grillon, C., \& Davis, M. (2001). Activation of the left amygdala to a cognitive representation of fear. Nature Neuroscience, 4, 437-441

Pizzagalli, D. A., Greischar, L. L. \& Davidson, R. J. (2003). Spatiotemporal dynamics of brain mechanisms in aversive classical conditioning: High-density event-related potential and brain electrical tomography analyses. Neuropsychologia, 41, 184-194.

Potts, G. F., \& Tucker, D. M. (2001). Frontal evaluation and posterior representation in target detection. Cognitive Brain Research, $11,147-156$
Rösler, F., Heil, M., Bajric, J., Pauls, A. C., \& Hennighausen, E. (1995). Patterns of cerebral activation while mental images are rotated and changed in size. Psychophysiology, 32, 135-149.

Rösler, F., Heil, M., \& Röder, B. (1997). Slow negative brain potentials as reflections of specific modular resources of cognition. Biological Psychology, 45, 109-141.

Sabatinelli, D., Bradley, M. M. Fitzsimmons, J. R. \& Lang, P. J (2005). Parallel amygdala and inferotemporal activation refiect emotional intensity and fear relevance. Neurolmage, 24, 1265-1270.

Schupp, H. T., Cuthbert, B. N., Bradley, M. M., Cacioppo, J. T., Ito, T. \& Lang, P. J. (2000). Affective picture processing: The late positive potential is modulated by motivational relevance. Psychophysiology $37,257-261$

Schupp, H. T., Flaisch, T., Stockburger, J., \& Junghöfer, M. (2006) Emotion and attention: Event-related brain potential studies. Progress in Brain Research, 156, 31-51.

Schupp, H. T., Junghöfer, M., Weike, A. I., \& Hamm, A. O. (2003) Emotional facilitation of sensory processing in the visual cortex. Psychological Science, 14, 7-13.

Schupp, H. T., Öhman, A., Junghöfer, M., Weike, A. I., Stockburger, J. \& Hamm, A. O. (2004). The facilitated processing of threatening faces: An ERP analysis. Emotion, 4, 189-200.

Schupp, H. T., Stockburger, J., Codispoti, M., Junghöfer, M., Weike, A I., \& Hamm, A. O. (2007). Selective visual attention to emotion. Journal of Neuroscience, 27, 1082-1089.

Schupp, H. T., Stockburger, J., Schmälzle, R., Bublatzky, F., Weike, A I., \& Hamm, A. O. (2008). Visual noise effects on emotion perception: Brain potentials and stimulus identification. NeuroReport, 19, 167171.

Smith, J. C., Löw, A., Bradley, M. M., \& Lang, P. J. (2006). Rapid picture presentation and affective engagement. Emotion, 6, 208-214

Stolarova, M., Keil, A., \& Moratti, S. (2006). Modulation of the Cl visual event-related component by conditioned stimuli: Evidence for sensory plasticity in early affective perception. Cerebral Cortex, 16 $876-887$.

Thorpe, S., Fize, D., \& Marlot, C. (1996). Speed of processing in the human visual system. Nature, 381, 520-522.

Van Rullen, R., \& Thorpe, S. J. (2001). The time course of visual processing: From early perception to decision-making. Journal of $\mathrm{Cog}$ nitive Neuroscience, 13, 454-461.

Vuilleumier, P. (2005). How brains beware: Neural mechanisms of enotional attention. Trends in Cognitive Sciences, 9, 585-594.

Vuilleumier, P., Armony, J. L., Driver, J., \& Dolan, R. J. (2001). Effects of attention and emotion on face processing in the human brain: An event-related fMRI study. Neuron, 30, 829-841.

Vuilleumier, P., Richardson, M. P., Armony, J. L., Driver, J., \& Dolan, R. J. (2004). Distant influences of amygdala lesion on visual cortica activation during emotional face processing. Nature Neuroscience, 7 $1271-1278$. 